\title{
Registros de prensa de los conciertos de Antonio Jiménez Manjón, Miguel Llobet y Agustín Barrios en Chile en los albores del siglo XX
}

\author{
Press cuttings of guitar concerts by Antonio Jiménez Manjón, \\ Miguel Llobet and Agustin Barrios in Chile \\ at the beginning of the twentieth century
}

\author{
por \\ Pablo Soto Hurtado \\ Departamento de Música, \\ Universidad Metropolitana de Ciencias de la Educación, Chile. \\ pablo.soto@umce.cl
}

El presente artículo constituye una primera aproximación, basada en una pesquisa hemerográfica, a las actividades realizadas en Chile por los guitarristas Antonio Jiménez Manjón, Miguel Llobet y Agustín Barrios, entre 1893 y 1918. En el caso de Manjón, la positiva recepción de sus conciertos tensionó algunos discursos establecidos en el medio local que consideraban a la guitarra como vulgar e inexpresiva. Llobet, por su parte, dio a conocer en Chile la existencia de la denominada "Escuela de Tárrega" ya en 1912, lo que constituye un hito relevante para el estudio de dicha escuela en nuestro país. Asimismo, su técnica de ejecución fue elogiada y, así como en el caso de Manjón, permitió cuestionar los mismos prejuicios negativos. El caso de Agustín Barrios llama la atención, porque, hasta donde sabemos, no alcanzó la misma cobertura y el mismo éxito que sus predecesores, pese a ser un destacado guitarrista y compositor. La revisión de las actividades de estos artistas pone en evidencia la masiva presencia de discursos en torno a la guitarra, los que han ido configurando una particular manera de comprender el desarrollo del instrumento en nuestro país.

Palabras clave: Guitarra clásica en Chile, técnica de ejecución guitarrística, discursos acerca de la guitarra, Manjón, Llobet, Barrios.

Based on a hemerographic investigation, this article constitutes an initial look at events held by guitarists Antonio Jiménez Manjón, Miguel Llobet and Agustín Barrios in Chile between 1893 and 1918. In the case of Manjón, the positive reception his concerts received went against some of the discourse in the local media, which considered the guitar vulgar and expressionless. For his part, Llobet made the 'Tárrega School'known in Chile in 1912. This proved a relevant milestone in the study of that school in our country. His technique was praised and, as with Manjón too, led to a questioning of the prejudices against the guitar. The case of Agustin Barrios is noteworthy since, as far as we know, he did not gain the same recognition and success as his predecessors, even though he was an outstanding guitarist and composer. Going over the events held by these artists shows that negative discourse about the guitar was widespread, leading to a specific way of understanding the development of the instrument in our country.

Keywords: Classical guitar in Chile, guitar-playing technique, guitar discourse, Manjón, Llobet, Barrios. 


\section{INTRODUCCIÓN ${ }^{1}$}

En los albores del siglo XX se registró en Santiago, Valparaíso y otras ciudades del país, una importante actividad de conciertos de connotados guitarristas extranjeros. El conocimiento y registro de estas actividades, las que permanecían hasta ahora mayormente en el desconocimiento, viene a complementar los avances alcanzados por otras investigaciones en torno a la historia de la guitarra clásica en Chile. En particular, nos referimos a los importantes estudios realizados por Jaime Calisto (1996) y Christian Uribe (2003).

En este trabajo, junto con presentar la información recabada acerca de algunos ${ }^{2}$ de los conciertos brindados por estos guitarristas, presentamos también un breve análisis basado en la recepción que la prensa tuvo de aquellos artistas y sus conciertos. En ese sentido, comprobamos la persistencia de un discurso "adanista", el que asumía que el estreno de cada guitarrista en el país (al menos en el caso de Manjón y Llobet) representó siempre la "primera vez" en donde fue posible oír la guitarra ejecutada de una manera excepcional, "como nunca antes se ha visto". En segundo lugar, vemos la presencia generalizada de una visión negativa acerca del valor artístico y social del instrumento, así como de sus capacidades expresivas. Por último, notamos que la apreciación de la técnica de ejecución, así como de la performance $e^{3}$ de estos guitarristas, se encuentra notoriamente mediada por una retórica vinculada al pensamiento romántico del arte.

Con "adanismo" nos referimos a cómo el mito de lo primigenio es persistentemente utilizado para describir el impacto de los guitarristas extranjeros en nuestro país. Esta premisa se basa en la propuesta de Alejandro Vera (2014), quien afirma que una explicación mitológica del descubrimiento de América ha sido recurrentemente aplicada para dar cuenta de los diferentes acontecimientos y procesos en la historia musical chilena. Según Vera, dicho mito considera los siguientes supuestos:

“[...] la premisa del vacío cultural americano, según la cual la cultura europea habría sido impuesta donde no había otra; como consecuencia de ello, los habitantes originarios no pueden considerarse como verdaderos agentes en el proceso de incorporación de dicha cultura, sino únicamente como receptores pasivos del mismo, así como de todos los procesos posteriores de cambio cultural; y como corolario, la imposición de la cultura europea habría conllevado un efecto civilizador, permitiendo poner al día y, aun más, movilizar un territorio que anteriormente se encontraba prácticamente inerte y carecía de una verdadera historia” (Vera 2014: 8).

Como veremos más adelante, las ideas descritas anteriormente se encuentran implícitas en gran parte de las crónicas revisadas. Es recurrente leer frases como: "nunca antes había sido posible oír la guitarra ejecutada de un modo verdaderamente artístico" o, "que la técnica de ejecución de estos artistas no tiene precedentes". Estas impresiones nos hablan de un contexto guitarrístico nacional "carente de una verdadera historia”, es así como por

1 Este trabajo forma parte de una investigación doctoral titulada "La técnica de la guitarra clásica en el Chile del siglo XX. Aportes para una historia crítica", realizada por el autor en la Pontificia Universidad Católica de Chile bajo la tutela de Alejandro Vera. Una versión preliminar de este trabajo se presentó en el Congreso Latinoamericano de Investigación Musical Karl Bellinghausen, realizado en Ciudad de México entre el 10 y el 13 de septiembre de 2018.

2 Al tratarse de un estudio preliminar que aborda la actividad de tres artistas de una manera general, no hemos incluido la totalidad de conciertos brindados por estos guitarristas en nuestro país, porque la extensión del trabajo se ampliaría considerablemente. Por tal razón, queda abierta la posibilidad de profundizar en cada uno de ellos en trabajos posteriores. Esto aplica principalmente para el caso de Manjón y Llobet, quienes sabemos tuvieron una extensa actividad guitarrística en Chile.

3 En este caso, entendemos performance simplemente como la presentación pública del artista. 
la actividad de estos músicos extranjeros se pudo acceder al arte de la guitarra, equivalente casi a un exitoso proceso de modernización.

Esta percepción del contexto local se relaciona evidentemente con la presencia generalizada de una valoración negativa de la guitarra como instrumento de arte. Por esta razón, resultaba sorprendente comprobar que podía no solo ejecutar un repertorio culto europeo, sino que además conmover profundamente por medio de su ejecución.

Estos discursos negativos no eran en ningún caso nuevos y circunscritos únicamente a la realidad chilena. Como bien lo resume y explica Luis Achondo (2015), es perfectamente posible rastrear la persistencia de estas ideas, validadas por diferentes argumentos, incluso desde hace varios siglos. Alejandro Vera (2016), por ejemplo, señala que ya desde el siglo XVII la guitarra era considerada despreciativamente por la elite intelectual, quien la comparaba con la vihuela.

En el transcurrir de los años, otros argumentos confluyeron en esta construcción peyorativa. Por lo general, fluctuando entre la crítica al instrumento mismo (poca sonoridad, dificultad en la ejecución, repertorio vulgar y sencillo), y la asociación que ha tenido -o se le ha asignado- con segmentos populares de la sociedad (instrumento de "cantoras", de "taberna", de "borrachos", etc.). Ejemplo de esto lo podemos ver en la siguiente cita:

En la calle Cuyo esquina Montecaseros, existe una taberna donde noche a noche se reúne un gran número de atorrantes, los que, a son de órgano, acordeón y guitarra, pasan la noche entregados a jaranas que no dejan reposar en paz a ningún vecino (Otero 2010: 349-350).

En esa línea es que adquiere sentido la "neurosis"4 que ha existido entre diferentes guitarristas y compositores a lo largo del tiempo por revalorizar el instrumento y posicionarlo en un contexto artístico "superior". Por lo general, hasta hace algún tiempo no existía mucha discusión en atribuirle exclusivamente a Andrés Segovia el haber logrado dichos objetivos. Sin embargo, un examen más exhaustivo de este relato nos posiciona frente a una realidad mucho más compleja, en donde la acción de otros guitarristas ha quedado omitida. Como veremos en este estudio, por ejemplo, en Chile ya a inicios del siglo XX estuvimos en presencia de guitarristas que realizaron una intensa labor de difusión de la guitarra de concierto. Estos artistas, además, no solo aportaron a la promoción del instrumento, sino que ampliaron el repertorio con obras propias o arreglos, al tiempo que traspasaron sus conocimientos siendo destacados pedagogos. Adicionalmente, Llobet y Barrios fueron los primeros guitarristas que grabaron sus interpretaciones en la naciente industria discográfica (Hoke 2013).

La revisión del mito de Segovia como reformador de la guitarra, como bien lo realizó en profundidad Achondo (2015), puede entenderse en gran medida como una construcción discursiva llevada adelante por el mismo guitarrista, reforzada desde la crítica musical española, al alero de las políticas de expansión cultural nacionalistas promovidas por la dictadura franquista. Lo anterior, no desmerece según nuestra opinión la calidad y el tremendo aporte realizado por Segovia como guitarrista y promotor de la creación de nuevas obras. Más bien, nuestra intención es señalar que la "neurosis" de renovación es posible rastrearla desde hace varias décadas, debido a la visión despectiva que ha versado respecto

4 Gerardo Mosquera utiliza el concepto de "neurosis" para calificar la constante ansiedad que ha caracterizado a la cultura y al arte latinoamericano en la búsqueda de una identidad particular. Creemos que este impulso es comparable a la persistente necesidad que ha atravesado generaciones de guitarristas y compositores por posicionar a la guitarra como un instrumento artístico. Para más detalle, véase Mosquera 2010: 123-133. 
de la guitarra, y que ha sido trabajo de muchos guitarristas -no solo de Segovia- el haber alcanzado éxito en esta empresa.

Finalmente, con "retórica romántica" nos referimos a la presencia en las críticas de conciertos, de ideas vinculadas con el discurso acerca del arte y la música en el período romántico del siglo XIX. Entre estas, destaca la suposición de que la valía de un artista está mediada por su capacidad de "[...] asumir una nueva dimensión y adoptar una misión reveladora de verdades de otro modo inaccesibles al hombre" (Fubini 2004: 121). En este caso, vemos que el guitarrista alcanzaba el éxito principalmente cuando emocionaba al auditorio, pues se esperaba presenciar una interpretación conmovedora diferente a cualquier tipo de ejecución conocida. Para nuestro estudio, esta vinculación discursiva con el romanticismo adquiere un trasfondo mayor, frente a la negativa visión que se tenía del instrumento. Como veremos más adelante, el artista extranjero redime la guitarra de su pobre condición inicial para así transformarla en un instrumento superior, capaz de expresar y conmover. Esta construcción, que combina la retórica romántica que le asigna al artista una capacidad transformadora, con el establecimiento de una condición negativa inicial hacia la guitarra, es recurrentemente usada para describir el impacto de Manjón y Llobet.

Además, los artistas acá presentados son tratados como concertistas en "guitarra clásica", únicamente para diferenciarlos de otros guitarristas de la época vinculados también al ámbito de lo popular o folclórico, a las estudiantinas o a la música de salón ${ }^{5}$. Huelga decir que esta separación en ningún caso supone una valoración dispar de una manifestación por sobre otra, ni menos pretende establecer una teoría acerca de qué es o no la guitarra clásica, asunto que por cierto sobrepasa la extensión e intereses de este artículo. Esta diferenciación busca principalmente aclarar el panorama guitarrístico chileno de esa época para ofrecer un estudio ordenado.

Damos esta catalogación a los artistas acá estudiados por las siguientes razones: su repertorio de concierto por lo general no incluía música vinculada a las estudiantinas ni al ámbito del salón, como pequeñas danzas y canciones, sino que ya abordaba a los "clásicos" de la música y de la guitarra como Beethoven, Schumann, Mozart, Bach, y también Aguado, Arcas, Coste, Sor, Tárrega y Barrios, entre otros (ver Anexos 1, 2 y 3). Asimismo, sus presentaciones se realizaron por lo general en grandes teatros y salas de conciertos y no tanto en el ámbito privado, de igual forma, fueron ampliamente difundidas y comentadas por la prensa de la época como sucesos extraordinarios en la vida cultural local. Por lo demás, estos artistas estaban en posesión de una técnica de ejecución que la crítica calificaba como "superior" en comparación a la de la guitarra popular o folclórica.

Pese a no compartir esta valorización negativa implícita hacia estas manifestaciones locales, entendemos que lo que se quería decir es que estos artistas ejecutaban un repertorio musical que precisaba de una mayor preparación desde un punto de vista técnico, estilístico y de proyección y manejo del sonido.

Debido a esta referencia permanente a lo técnico que encontramos en las crónicas de conciertos, es que quisiéramos referirnos brevemente al concepto de técnica de ejecución musical, para así diferenciarlo del de escuela técnica, pese a ser un tema complejo que por cierto amerita una mayor discusión ${ }^{6}$. En este trabajo, entendemos la técnica como el conjunto de acciones e ideas que componen la ejecución musical. Estas acciones e ideas las vinculamos con tres dimensiones presentes en la ejecución de cualquier instrumento: lo mecánico, lo

5 Me refiero, por ejemplo, a guitarristas como Antonio Alba, Carlos Pimentel, E. J. Hermosilla, Francisco Rubí, Joaquín Zamacois, entre otros. Para mayor detalle de la actividad de estos artistas, véase Uribe 2003.

6 Para revisar otros textos que han abordado el tema de la técnica desde la perspectiva aquí discutida, véase, entre otros, Chiantore 2001, Johansson 2014 y Fernández 2000. 
discursivo y lo sonoro. Lo mecánico, refiere al ámbito corporal o físico involucrado en el acto de la producción del sonido (por ejemplo, el movimiento de las diferentes falanges de los dedos). Lo discursivo, es el ámbito encargado de construir, justificar y validar desde el lenguaje hablado todo el sistema en su conjunto (por ejemplo, "se toca de esta manera porque así la guitarra suena como el piano y no como una mandolina"). Por último, lo sonoro, es decir, el resultado estético particular derivado de los mecanismos utilizados en la ejecución (esta diferencia se percibe únicamente mediante el acto de escucha) ${ }^{7}$.

Entendemos que la presencia de una escuela técnica queda supeditada a la existencia de un método escrito que "[...] demuestre y sistematice las enseñanzas de un maestro" (Hernández 2010: 17); así como de una detallada teoría instrumental entendida como "[...] un conjunto orgánico lógicamente estructurado de soluciones a cada uno de los problemas que plantea la mecánica del instrumento" (Escande 1996: 2). Lo anterior promueve, al menos en teoría, una base sólida sobre la que podría establecerse una uniformidad respecto de los aspectos mecánicos, discursivos y sonoros de la ejecución musical.

Dicho esto, revisemos la actividad de Jiménez Manjón, Miguel Llobet y Agustín Barrios en nuestro país.

\section{ANTONIO JIMÉNEZ MANJÓN, "UN GUITARRISTA COMO NINGÚN OTRO”}

El debut del guitarrista Antonio Jiménez Manjón en nuestro país lo registramos a fines de noviembre de $1893^{8}$, en un Teatro Municipal desbordante, donde "[...]las más distinguidas damas de la sociedad santiaguina orlaban los palcos y la platea semejaba florido jardín de primavera" ${ }^{9}$. Tal expectación por escuchar al guitarrista se explicaba en gran medida por la reputación internacional que lo precedía: “[...] su competencia en la guitarra había sido reconocida por los públicos más ilustrados del mundo y por lo tanto era justa la curiosidad de escucharle a causa de su celebridad" 10 .

El éxito inicial alcanzado en Santiago se repitió en Valparaíso el 22 de diciembre de 1893, pese a que la asistencia de público fue notoriamente menor que en la capital. Esto último se entendería, de acuerdo con la cita del periódico, por la apreciación despectiva de la elite del puerto hacia el instrumento:

Una concurrencia apenas regular asistió anoche al estreno del guitarrista señor Manjón. Brilló por su ausencia la high life porteña, sin duda por sonarle mal al oído eso de la guitarra ¿Qué más si hubieran sabido que era guitarrón, y tocado por un ciego! [...] En cambio los que concurrieron no deben haberse arrepentido, porque el guitarrista señor Manjón, lo mismo que su esposa Salazar, cumplieron el programa y justificaron su reputación artística de una manera brillante [...] No hubo parte del programa que no llamase la atención, ya tocase solo el eximio guitarrista, o ya acompañado de su esposa, quien también se hizo aplaudir y admirar en las partes que ejecutó

7 Para una discusión mayor de este tema, véase Soto 2018a.

8 La visita de Manjón a nuestro país fue informada previamente por González y Rolle 2005. En este trabajo, los autores señalan lo siguiente: "Ese mismo año [1894] la guitarra elevaba su categoría artística en el país con la visita del guitarrista clásico español Antonio Jiménez Manjón [1866-1919], con el consiguiente incremento de su prestigio social para las elites". Véase González y Rolle 2005: 60.

9 Re-La-Mi-Do "Teatro Municipal”, El Noticiero Español. Periódico Semanal Independiente. Dedicado a la Colonia Española, II/70 (30 de noviembre, 1893), p. 3.

10 Re-La-Mi-Do "Teatro Municipal”, El Noticiero Español. Periódico Semanal Independiente. Dedicado a la Colonia Española, II/70 (30 de noviembre, 1893), p. 3. 
ella sola en el piano. Sin embargo, buenos pianistas hemos oído muchos, pero un guitarrista como el señor Manjón, ninguno ${ }^{11}$.

Si bien no sabemos con exactitud cuánto tiempo vivió en Chile el guitarrista, podemos concluir que su estadía fue prolongada, ya que sabemos que en nuestro país nacieron sus tres primeros hijos (Ramos 2009: 1). Esta hipótesis se refuerza, además, a partir de la información brindada por la prensa de sus conciertos en Valparaíso veinticinco años después de su llegada al país, en 1918. En esta se enfatiza que el artista es reconocido en el medio local: "[s] egún hemos dicho, esta noche se presenta al público, en el salón concierto del Centro Español -calle Victoria, pasado Avenida Francia- el reputado guitarrista español, señor Manjón, conocido ya de nuestro público"12.

Lo mismo se desprende de los comentarios de prensa a los intérpretes nacionales José Pavez Rojas y Esther Martínez. En el caso de la guitarrista, las críticas a su desempeño artístico señalan al concertista español como un referente: "[s]i Antonio Manjón oyera a Ester ${ }^{13}$ Martínez, es seguro que el sublime ciego querría verla a través de sus ojos sin luz" 14 . Por su parte Pavez declara en un aviso de clases de guitarra inserto en Revista Música en 1920 (ver Figura 1), que realizó estudios con Manjón a modo de referencia y respaldo de su experticia y conocimiento. Estos datos corroborarían que su estadía en Chile fue extensa, porque era un artista reconocido y que gozaba de reputación en el medio local.

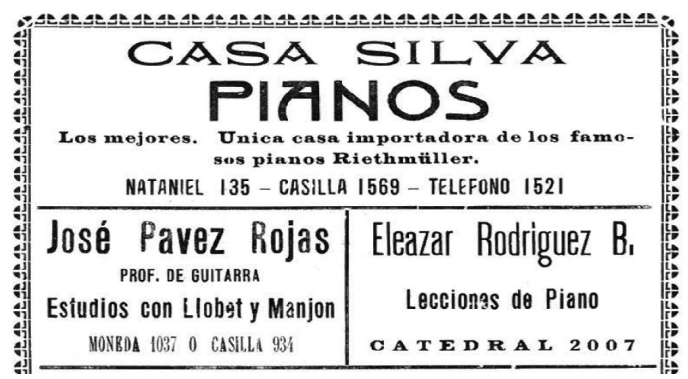

Figura 1. Anuncio de clases de guitarra de José Pavez Rojas. Revista Música. II/2 (febrero, 1920), p. 13.

Como dijimos, los conciertos de Manjón tensionaron esta impresión de la guitarra como un instrumento inexpresivo, imposibilitado de ejecutar una música culta de arte capaz de conmover y transmitir emociones:

Es necesario oírlo para esplicarse [sic] el poder de su ejecución, su manera de interpretar y la facilidad con que saca partido de un instrumento que parece sordo y refractario a todo sentimiento artístico. Tanto es así, que a veces uno se imajina [sic] no estar oyendo una guitarra o guitarrón, sino una orquesta con toda la dulce armonía de sus variados instrumentos ${ }^{15}$.

11 El Mercurio. Valparaíso, LXVII/19943 (23 de diciembre, 1893), p. 2.

12 El Mercurio. Valparaíso, XCI/28590 (21 de julio, 1918), p. 5.

13 En todas las referencias que hemos leído de la guitarrista, su nombre aparece como "Esther". Sin embargo, en esta crónica en particular aparece sin la "h". Por tanto, creemos que es un error de la fuente.

14 Revista Música, III/8 (1922), p. 4.

15 El Mercurio. Valparaíso, LXVII/19943 (23 de diciembre, 1893), p. 2. 
También es interesante observar la manera en que la prensa se refiere a la técnica de ejecución guitarrística a propósito de los conciertos de Manjón, y posteriormente como veremos de Llobet y Barrios. La descripción y valoración de la técnica es un ejercicio que ha estado presente en la crítica a lo largo del siglo XX, pero que no necesariamente se ha tratado con el mismo lenguaje y enfoque al que hoy estamos habituados. Esto nos permite conocer las diferentes visiones que se ha tenido en torno al tema. Por ejemplo, una perspectiva más pragmática, preocupada de destacar la exactitud de los movimientos y la cualidad de los timbres, no representa la época de fines del siglo XIX y principios del XX, en donde los principales criterios con que se relaciona la técnica son el nivel de dominio que se tiene del instrumento y con la capacidad de conmover y emocionar al público ${ }^{16}$.

Esto se verifica con Manjón, ya que el principal logro de su técnica es que puede dominar y ejecutar con pulcritud un instrumento difícil, "sordo" y "refractario", y que en esa acción es capaz de transmitir emociones y conmover:

Es asombrosa su ejecución, es inimitable su limpieza, es muy notable su inspiración y el gusto que sabe imprimir a las obras que interpreta; el auditorio se halla pendiente de sus dedos y ni el más insignificante ruido viene a perturbar el silencio religioso con que es escuchado; se necesita haber nacido artista para dominar la guitarra en la fortuna que lo hace el Sr. Manjón ${ }^{17}$.

En esa misma línea, según señala Prat en su famoso diccionario, Manjón fue esencialmente un guitarrista autodidacta, a pesar de que estudió con un discípulo de Aguado y "[...] que era gran amigo de la escuela de su maestro" (Prat 1934: 191). Es decir, recibió lecciones vinculadas con la Escuela de Dionisio Aguado, lo que al parecer complementó con otros aprendizajes adquiridos de manera más autónoma. Este dato nos parece importante de relevar en este estudio por dos razones. En primer lugar, no era un guitarrista que aparecía vinculado con la Escuela de Tárrega -tal vez la de mayor popularidad entre los guitarristas de esta época-, sino más bien con la tradición técnica más antigua. Sin embargo, y a diferencia de lo que veremos en las crónicas a Llobet en 1912 y 1918 -en las que sí se hacen menciones concretas a Tárrega como maestro y se presenta ya la idea de su escuela-, en el caso de Manjón no hallamos referencias a Aguado o a su propuesta técnica en sus apariciones en la prensa chilena. Por esta razón, en términos de la recepción en nuestro medio de la técnica de ambos guitarristas, hay una diferencia fundamental: en Llobet, era patrimonio de una escuela y un maestro: Tárrega; en el caso de Manjón, residía más bien en sí mismo como una invención o habilidad personal.

En segundo lugar, debemos recordar que una de las características centrales de la propuesta de Aguado era el uso de la pulsación con uña, al contrario de Fernando Sor, quien planteaba una ejecución con yema. Al parecer, esto último era lo más común porque el uso de la uña se vinculó, entre otras cosas, con una interpretación musical preocupada más bien por el virtuosismo, en tanto facilitaba la velocidad en la mano derecha (Pujol 1934).

Esta última apreciación es coincidente con las crónicas revisadas, pues se enfatiza que el público permanecía "cautivo" del movimiento de sus dedos, lo que seguramente refleja una ejecución muy virtuosa. Esto se confirma, además, por medio del repertorio

16 Para mayor detalle de esta información, véase Soto 2018a.

17 Re-La-Mi-Do "Teatro Municipal”, El Noticiero Español. Periódico Semanal Independiente. Dedicado a la Colonia Española, II/70 (30 de noviembre, 1893), p. 3. 
ejecutado en sus conciertos en nuestro país (ver Anexo 1), en donde se observan obras que requieren de un desarrollo de la habilidad y el virtuosismo, como el Rondó en La menor de Aguado. Esta obra exige una gran destreza en el uso de arpegios y escalas, las que seguramente resultarían muy difíciles de realizar pulsando con yema.

Además, es sabido que Manjón ejecutaba una guitarra ampliada de once cuerdas, también conocida a veces como guitarrón. Según Ignacio Ramos, este modelo no era del todo novedoso, ya que varios otros guitarristas españoles lo ocupaban, "[...] como García Tolsa, José Rojo, Luis Soria y Martínez Toboso”. Asimismo, continúa Ramos, fue utilizada por varios de sus discípulos en Argentina, “[...] como Emilio Bo y su hijo César, Rómulo Troncoso, Yolanda Grasso o Margarita Gachitegui, y algún brasileño como Alfredo de Souza-Iménez" (Ramos 2005: 120).

Debemos recordar que estamos en un momento en el que se estaban desarrollando innovaciones en la manufactura del instrumento, pues existía, entre otras razones, una aspiración por encontrar un modelo que facilitara la inclusión de la guitarra en el ámbito de concierto internacional. Desde siempre la escasa proyección sonora alcanzada por el instrumento coartó la participación de guitarristas en las grandes salas y teatros de Europa y América. En este contexto de transformación, la guitarra ampliada de Manjón, e incluso otras experimentaciones más osadas como la "guitarra-arpa" de Pedro Dorrego y Luis Soria ${ }^{18}$, resultan comprensibles desde el punto de vista de la experimentación.

En el caso de sus actuaciones en Chile, hasta donde sabemos, en ninguna de las crónicas se señalan mayores detalles acerca de su guitarra de once cuerdas, o del impacto que este instrumento pudo haber provocado en el público local.

\section{MIGUEL LLOBET Y LA ESCUELA DE TÁRREGA EN CHILE}

Los conciertos de Llobet en nuestro país fueron largamente anunciados y comentados por la prensa de la época (ver Figuras 2 y 3), por lo que suponemos que sus dos visitas, en 1912 y 1918 respectivamente, fueron verdaderos sucesos en el medio local. Probablemente, la expectativa por oírle se explique, al igual que en el caso de Manjón, por la extraordinaria crítica europea que lo precedía, la que lo presentaba como "el más célebre guitarrista del mundo", "el mejor guitarrista del mundo", o como "el primer guitarrista del mundo". En su primera gira se presentó en Santiago, Valparaíso y Concepción; mientras que en 1918 agregó las ciudades de Talca y Viña del Mar.

18 Dorrego fue un guitarrista argentino que brindó conciertos en Valparaíso y Santiago a inicios de 1860. Fue reconocido por utilizar un instrumento denominado "guitarra-arpa" que, como su nombre lo señala, consistía en la unión de una guitarra y un arpa. Un instrumento similar fue utilizado también por Luis Soria. Para mayor detalle, véase Ramos 2012: 25. 


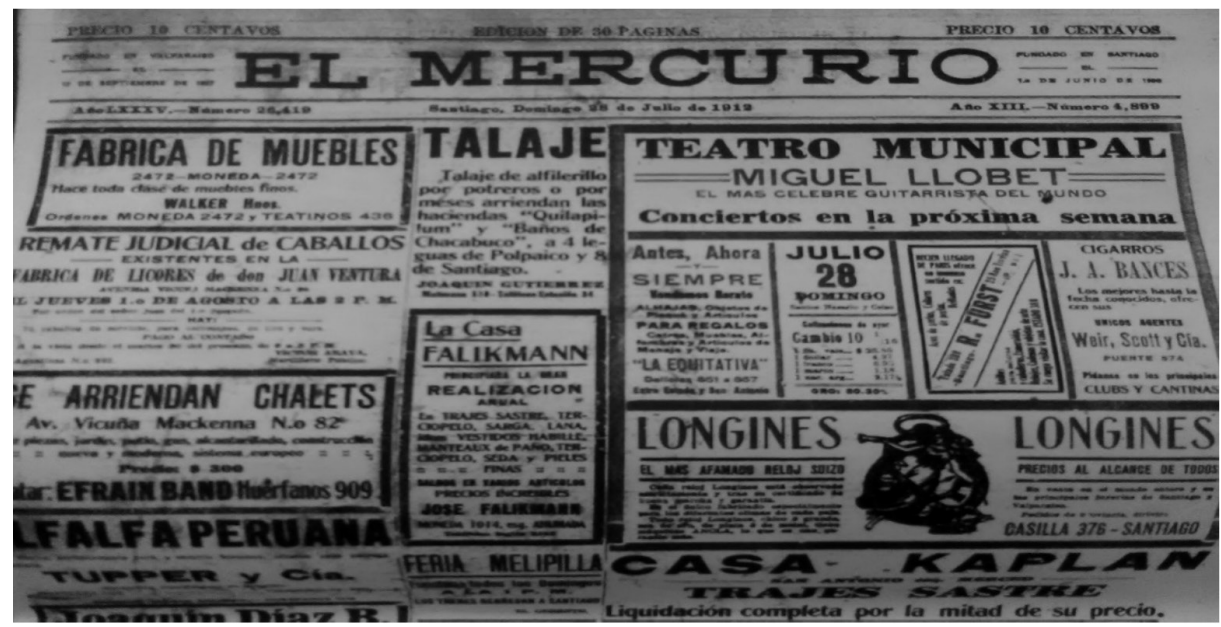

Figura 2. Anuncio de concierto de Miguel Llobet en el Teatro Municipal de Santiago. El Mercurio. Santiago, 28 de julio, 1912.

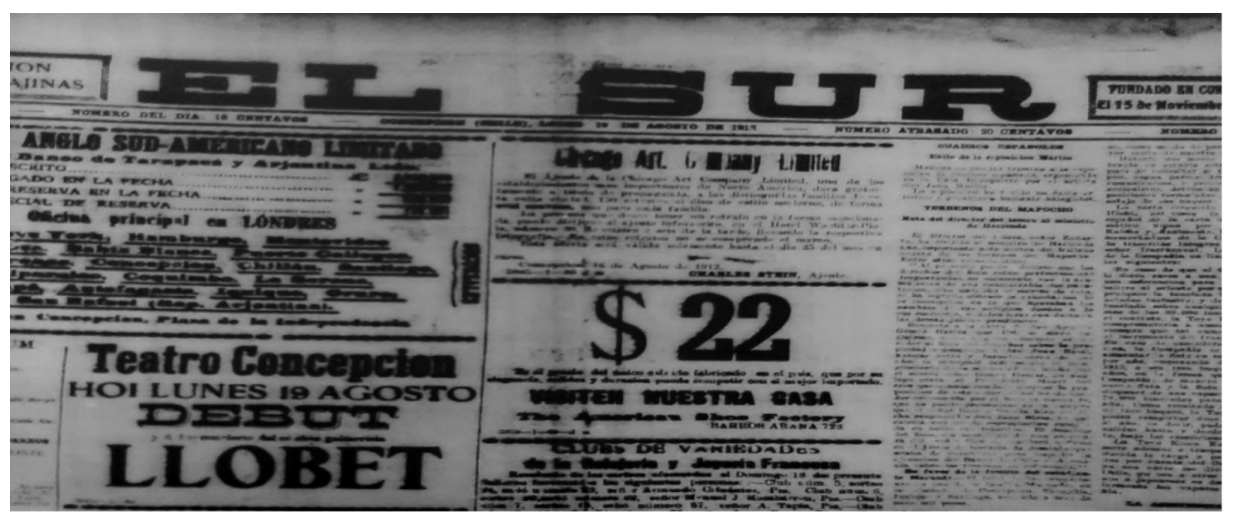

Figura 3. Anuncio de concierto de Miguel Llobet en Concepción. El Sur. Concepción, 19 de agosto, 1912.

Sus presentaciones permitieron cuestionar los mismos discursos que ya habían sido puestos en tensión hace casi veinte años. Por ejemplo, la prensa lamenta profusamente que Llobet con su tremendo talento se dedique “[...] al estudio de un instrumento que sin duda no ofrece el campo que otros para desarrollar en toda su extensión una inteligencia y un temperamento de gran artista" 19 .

La siguiente cita a propósito de un concierto en Valparaíso en 1918, nos presenta de manera precisa dos de los elementos discursivos que hemos revisado en este estudio. Por una parte, la visión negativa del instrumento y, por otra, la capacidad transformadora del artista que redime al instrumento "vulgar, generalmente despreciado", para posicionarlo

19 "El guitarrista Llobet”, El Mercurio. Santiago, LXXXV/26429 (7 de agosto, 1912), p. 14. 
junto con otros que, según el criterio de la época, conformaban la denominada "aristocracia instrumental":

La guitarra, instrumento popular, cuyos rasgueos, especialmente entre nosotros, apenas si sirven para acompañar a la cantadora que entona una 'tonada' con voz de falsete, ha perdido hace muchos años el prestigio de que gozara en otras épocas. Y así, cuando aparece un Tárrega, un Llobet, que la pulsan y le arrancan sus más vibrantes armonías, la guitarra parece revivir, y el auditorio, bajo la sugestión del artista, ve en ella, no el instrumento vulgar generalmente despreciado, sino un instrumento nuevo, digno de figurar junto a aquellos que constituyen la aristocracia instrumental: el piano, el violín y el violoncelo, puesto que todos ellos tienen la armonía y la suprema gracia ${ }^{20}$.

No se escatima en elogios, además, para referirse a la ejecución técnica de Llobet, destacando su dominio sobre el instrumento y el gran nivel de dificultad y belleza del repertorio interpretado (ver Anexo 2). A partir de una retórica romántica, que ya hemos discutido, se describe cómo con la profundidad de su interpretación conmueve intensamente al auditorio: "[...] mientras sus dedos vuelan sobre las cuerdas, los ojos se cierran y se ve entre velos azules, paisajes lejanos [...] y luego un alma que languidece, que suspira" 21 . Se enfatiza que es un ejecutante virtuoso de mucha destreza y habilidad: "[...] la guitarra, bajo sus dedos, adquiere vida propia"22. También, se subraya su capacidad de obtener variados recursos sonoros y tímbricos en un "instrumento como ese", por lo que "[1]a nitidez de la mecánica y el colorido propio de todo un mago del arte, formaban los conceptos más acertados y unánimes en el público de anoche"23. En suma, la técnica de Llobet es descrita en forma general de esta manera:

La técnica del artista es insuperable: agilidad, finura dentro de esta misma agilidad y precisión del maestro. Destacaban tan claramente el canto y el acompañamiento en su ejecución que hubiérase [sic] podido decir que no era una sino dos las guitarras que, en ese momento, dejábanse oír. El señor Llobet pone en juego recursos preciosísimos: prolongación de sonidos, tonalidades las más diversas que casi fuera increíble que pudieran salir de un instrumento como ese ${ }^{24}$.

A lo anterior podemos agregar otros antecedentes para esbozar una idea más completa de la técnica de Llobet. Si observamos una fotografía ${ }^{25}$ del guitarrista durante su juventud (ver Anexo 8), notamos que su posición se asemeja bastante a la tradicionalmente asignada a Tárrega (ver Anexo 9): hombro derecho inclinado hacia adelante; el brazo izquierdo más contenido, pegado al cuerpo con la muñeca hacia adentro; y, por último, la mano derecha atacando en forma perpendicular a las cuerdas. Como sabemos, Llobet estudió con Tárrega a partir de 1894, al parecer sin basarse en una metodología particular, sino más bien observando y experimentando con su técnica en lugar de aprenderla fielmente (Phillips 2002: 10). Esto podría explicar las semejanzas anteriores y, a la vez, la diferencia entre el ataque con uña de Llobet en desmedro de la pulsación con yema de Tárrega. Esto último configura una separación entre la técnica del maestro y su discípulo, y permite posicionar

20 L.E.C, "El concierto Llobet”, El Mercurio de Valparaíso, XCI/28592 (23 de julio, 1918), p. 3.

21 Rafael Coronel, "La audición íntima de Llobet", Las Últimas Noticias, Santiago, XVI/4799 (13 de julio, 1918), p. 3.

22 L.E.C, "El concierto Llobet", El Mercurio de Valparaíso, XCI/28592 (23 de julio, 1918), p. 3.

23 Juan Filippi Dagnino, "Llobet", El Mercurio de Valparaíso, LXXXV/26421 (13 de agosto, 1912), p. 4.

24 "El guitarrista Llobet”, El Mercurio. Santiago, LXXXV/26429 (7 de agosto, 1912), p. 14.

25 Sabemos que las fotografías de los guitarristas y artistas de la época correspondían en ocasiones a referencias publicitarias. Esto quiere decir que estamos al tanto de la complejidad de arribar a conclusiones únicamente a partir de estas fuentes, por lo que asumimos que pueden ser discutibles. Sin embargo, en este caso, la postura analizada de Llobet y de Tárrega que se describe en sus fotografías, cuenta además con testimonios que corroboran tal descripción. Véase Prat 1934. 
a Llobet como uno de los guitarristas que más contribuyó a la promoción de ese criterio mecánico -ataque con uña- en el siglo XX (Phillips 2002: 102).

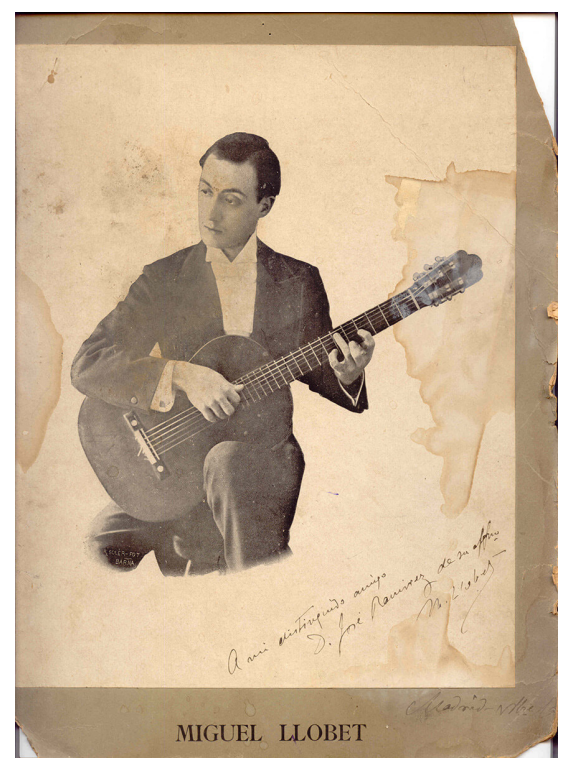

Figura 4. Fotografía de Miguel Llobet en su juventud ${ }^{26}$.

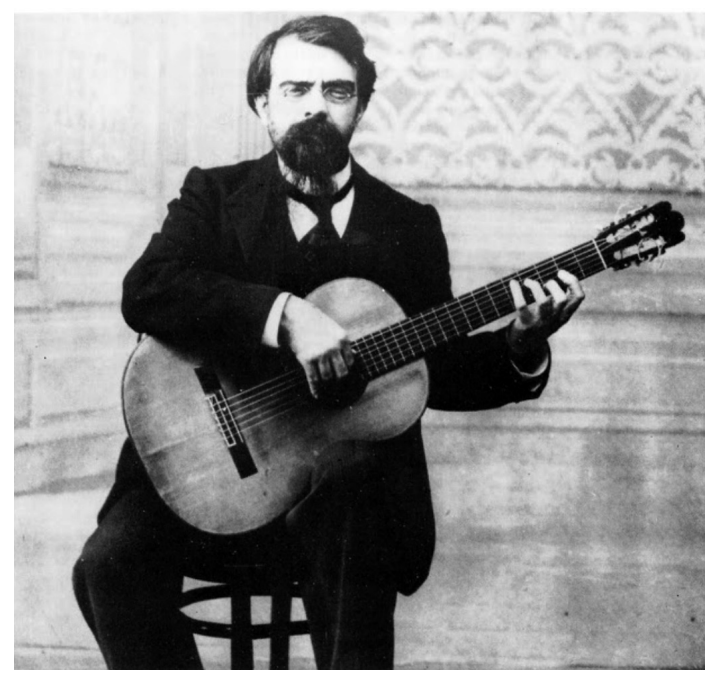

Figura 5. Fotografía de Francisco Tárrega 27.

${ }^{26}$ Fuente: https://goldtonemusicgroup.com/img/joseramirez/museum/MIGUEL-LLOBET. jpg [acceso: 30 de noviembre de 2020]

27 Fuente: https://elmiradornocturno.blogspot.com/2013/11/francisco-tarrega.html [acceso: 30 de noviembre de 2020] 
Independiente de esta diferencia -y más importante para este estudio-, suponemos que a partir de los conciertos de Llobet se conoció por primera vez de forma masiva la figura de Tárrega y la existencia de su escuela en nuestro país ${ }^{28}$. Resulta interesante notar, entonces, la forma en la que se explicaba por esa fecha en qué consistía dicha escuela ${ }^{29}$ :

Fárrega [sic], eminente concertista, ha sido el evolucionador, el revolucionario que estimando en todos sus méritos a Sor y otros geniales de la guitarra, respetó hasta donde fue posible teorías algo añejas, pero introdujo reformas saludables en el estudio de que Llobet es fanático admirador. Desde luego la escuela de Llobet es la escuela de Fárrega [sic], aquella que acepta reformas en el estudio, pero no en el instrumento. Según estos principios autorizados las seis cuerdas bastan para alcanzar el máximum de sonoridad y efecto. Agregar mayor número es variar la forma y detalles de construcción que pecan en su base por tratarse de obtener lo que en su origen no se creyó necesario y siguen sosteniéndolo artistas de talla mundial ${ }^{30}$.

Aparte del evidente error ortográfico podemos comentar varias otras cosas. En primer lugar, que el maestro catalán es presentado en una línea histórica que viene directamente desde Sor "y otros geniales de la guitarra", por tanto, no es descrito como una figura aislada o rupturista, sino ligado a la tradición. En segundo lugar, se percibe ya desde este momento una ambigüedad con relación a cuáles fueron los cambios concretos en la mecánica de la técnica introducidos por Tárrega. Acá, por ejemplo, se señala únicamente que fueron "reformas saludables" pero no hay un detalle mayor. Es posible notar que esta falta de claridad la veremos en la mayoría de las descripciones de su escuela (Soto 2018a). En tercer lugar, se reafirma lo discutido anteriormente en cuanto se asiste a un período de transformación en la manufactura del instrumento. En esa línea, se muestra seguramente una discrepancia con Jiménez Manjón y su guitarra de once cuerdas ${ }^{31}$.

Profundizando aún más en este último punto, sabemos que el constructor Antonio Torres Jurado fue quien estableció de manera general los cánones de construcción y diseños básicos de la actual guitarra de concierto. Entre otras cosas, perfeccionó el " [...] sistema de refuerzo con varas de madera en abanico que se adosaban a la parte inferior de la tapa armónica para conseguir una mejor proyección del sonido" (Ramos 2005: 130). También aumentó el tamaño de la caja de resonancia y de la anchura del mástil, estableció las longitudes del traste y el tiro de las cuerdas, y promovió el uso de maderas y barnices de primera calidad (Ramos 2005: 130).

Estas innovaciones facilitaron la entrada del instrumento a las grandes salas y teatros, lo que benefició a guitarristas como Llobet, que logró desarrollar una carrera de concertista

28 Aparte de la cita presentada en el texto principal, encontramos varios otros ejemplos en la prensa nacional que vinculan a Llobet con Tárrega como su maestro, solo por citar dos: "Llobet fue preferido alumno del notable compositor Tárrega, de quien en la actualidad es dignísimo sucesor" ("Miguel Llobet", El Sur, Concepción, XXXV/13155, [3 de agosto, 1918], p.4); "Cuando Llobet hubo terminado sus estudios generales se puso en manos del gran Tárrega, célebre maestro valenciano, que verificó su completa educación en la guitarra" ("Hoy el guitarrista Llobet en el Palet", La Mañana, Talca, XII/3917, 7 de agosto, 1918, p. 3).

29 Recordemos que cuando Llobet visitó Chile por primera vez, en agosto de 1912, recién habían transcurrido dos años y medio aproximadamente de la muerte de Tárrega, por lo que la idea de su escuela estaba en pleno proceso de formación.

30 Juan Filippi Dagnino, Sucesos de Valparaíso, XI/ 520 (22 de agosto, 1912) p.16.

31 A pesar de no ser una discrepancia directa, la idea de que no se deberían hacer reformas al instrumento también afectará la apreciación del medio a los conciertos de Agustín Barrios, quien tocaba con cuerdas metálicas. Como veremos, se le criticó fuertemente que con esa elección se alejaba de la tradición original de la guitarra. 
en parte por estas mejoras sustanciales. De esta forma, no extraña que en algunas notas de prensa se aborde este tema, pues, al parecer, su guitarra Torres causó una gran impresión en el medio local.

-Hablemos de su guitarra. ¿De dónde procede?

-Mi guitarra es una 'Torres' auténtica. Torres fue un ebanista andaluz que se especializó en la fabricación de estos instrumentos. Era una especie de Stradivarius de la guitarra.

-A propósito de Tárrega: hace años, usted anduvo empeñado en comprar una guitarra que perteneció al maestro.

-Exacto. Quería conservar ese recuerdo; pero no me fue posible. La adquirió una dama argentina, en una suma crecidísima: 4 o 5 mil pesetas ... Era una 'Torres' como la mía. ${ }^{32}$

Volviendo a la difusión de la Escuela de Tárrega en Chile, es destacable reconocer que sea posible situar en 1912 un hito inicial. Esto permite concluir, entre otras cosas, que algunas de las versiones posteriores de esta escuela -como la de Albor Maruenda o Liliana Pérez Corey- no fueron las únicas ni las primeras que existieron en el medio local.

Cada una de estas versiones -o apropiaciones y lecturas personales de la escuela- tuvieron un desarrollo diferente en el transcurso del siglo XX (Soto 2018a). En el caso de Llobet -o lo que podríamos denominar Escuela de Tárrega/Llobet-, a pesar del tremendo impacto que provocó su figura en nuestro país, su trascendencia en términos pedagógicos es marginal y escasa. No vemos, como en otros países de la región, una línea reconocida de discípulos ${ }^{33}$ que hayan continuado su tradición, ya que, salvo el caso de José Pavez, no tenemos hasta ahora noticias de otros guitarristas chilenos que hayan estudiado con él. Y aunque los hubiera, no tuvieron connotación, al menos de manera oficial, pues no tuvieron participación en el Conservatorio Nacional.

Con el establecimiento del guitarrista argentino-español Albor Maruenda ${ }^{34}$ como profesor de guitarra del Conservatorio en 1938, se incorporó como modelo pedagógico la escuela de Tárrega en la línea vinculada con Josefina Robledo, quien, como sabemos, fue su maestra en Valencia ${ }^{35}$. En su trabajo en Chile, Maruenda recurrió a los métodos que hace poco había publicado Pujol, pues, según él mismo afirmaba, esta "[...] obra ponía en vigencia los planteamientos modernos de la guitarra, realizados por la labor ejemplar, abnegada, de Francisco Tárrega, su música y la labor de sus alumnos" (Maruenda 1996) ${ }^{36}$. No obstante lo discutible que resulta aseverar que La Escuela Razonada de Pujol sintetice los postulados técnicos de Tárrega ${ }^{37}$, la cita de Maruenda es relevante, pues nos dice que,

32 L.E.C, "Miguel Llobet”, El Mercurio de Valparaíso, XCI/28595 (29 de julio, 1918), p. 3.

33 Por ejemplo, María Luisa Anido en Argentina, e Isaías Savio en Uruguay.

34 Para más información respecto de Albor Maruenda, véase Soto 2018b.

35 Es complejo asumir de entrada el hecho de que solo por haber estudiado con tal o cual maestro el discípulo replique exactamente los mismos postulados pedagógicos. Entendemos que los conocimientos son dinámicos y que varían de persona a persona. Lo que queremos decir acá tiene que ver más bien con una cierta secuencia histórica que permite vincular a los guitarristas a partir de su punto de formación. Esto, creemos de una u otra manera, nos provee de un marco que visualiza la continuidad y proyección de ciertos maestros en esta etapa temprana chilena.

36 Carta inédita de Albor Maruenda a Jaime Calisto fechada el 15 de enero de 1996. Este documento se encuentra citado en Soto 2018a.

37 Existe una controversia respecto de la correspondencia entre el texto de Pujol y los postulados técnicos de Tárrega, pues, por más que Pujol se esfuerce en presentar su texto como la síntesis de la obra técnica de su maestro, en definitiva, resulta más bien como su propia obra técnica escrita bajo la figura de Tárrega. En cierta medida, esta vinculación funcionó como un importante argumento de validación de su método (para más detalle de esta controversia, véase: Hernández 2010). 
desde el inicio de la cátedra de guitarra en la academia nacional, este método fue utilizado como recurso pedagógico, lo que nos informa acerca de su impacto y proyección.

Bajo estos principios se formó Liliana Pérez Corey, una de las figuras de mayor connotación en la formación de guitarristas en Chile en la segunda mitad del siglo XX. Sin embargo, más allá de su contacto con el método de Pujol a la luz de las enseñanzas de Maruenda, resulta complejo establecer otros puntos de cercanía con la denominada Escuela de Tárrega/ Pujol. Como hemos revisado en otros trabajos (Soto 2018a), el vínculo de Liliana Pérez como estudiante de Pujol ha sido muy difícil de confirmar a partir de documentos. Esto nos llama la atención, por los extraordinarios relatos (Rodríguez 2001) acerca de la relación entre ambos guitarristas. En virtud de aquello, su establecimiento como continuadora de la Escuela de Tárrega/Pujol queda para nosotros como un tópico abierto para el debate y la profundización en posteriores investigaciones.

Además, tenemos noticias de que el guitarrista chileno Luis López también realizó estudios de perfeccionamiento con Pujol participando, al menos hasta donde sabemos, en un curso con el guitarrista en 1969 (Véase Hernández 2010: 206).

Hasta este punto podríamos definir la proyección directa de esta escuela con los guitarristas chilenos. Evidentemente este es un tema que precisa de un desarrollo mayor que excede el espacio de este estudio.

Con todo lo anterior, sabemos que Llobet contribuyó enormemente al conocimiento público de la obra y la figura de Francisco Tárrega en Chile, y jugó un rol destacado en la entrada y consolidación de la guitarra como instrumento de concierto en el contexto de la música docta. Su ingente cantidad de giras en un momento de difícil traslado entre países y continentes, además de su labor como profesor, le merece un espacio de reconocimiento que no siempre ha sido otorgado, sobre todo en comparación con otros guitarristas como Andrés Segovia o el propio Pujol.

\section{AGUSTÍN BARRIOS, "UN LLOBET AL ALCANCE DE LOS NIÑOS”}

Tenemos noticias de que el guitarrista y compositor paraguayo Agustín Barrios brindó al menos dos conciertos en el Teatro de la Unión Central de Santiago en noviembre de 1913. La prensa local lo presentó como un virtuoso de la guitarra que había alcanzado un importante reconocimiento en sus presentaciones por Sudamérica; destacando, particularmente, su dominio técnico sobre el instrumento:

Se nos dice que Barrios ha consagrado todas sus energías al dificilísimo estudio del clásico instrumento hispano y merced a su tenaz perseverancia, llegó a desentrañar los innumerables y complejos secretos de su técnica, haciéndose dueño de la caja armónica de la guitarra, inaccesible a casi la totalidad de sus cultores ${ }^{38}$.

A pesar de las loas previas a su presentación en Santiago, los comentarios posteriores a sus conciertos no fueron del todo felices. En parte, porque se apeló a algunos criterios de juicio diferentes a los que hemos visto anteriormente, como la particular mención al origen hispano del instrumento y, por esta razón, a la ligazón "natural" que supuestamente existiría entre los españoles y la guitarra:

38 "El guitarrista Barrios", El Diario Ilustrado, XII/4101 (8 de noviembre, 1913), p. 8. 
Es necesario que por las venas cruce mucho de sangre española [...] para consagrar un culto [...], una vida entera de arte y de esperanzas, al único, tal vez, de los instrumentos que se juzgaba condenado, en su abandono, a no encender jamás la flama del entusiasmo artístico en los públicos modernos, al único excluido de por sus condicionantes poco armónicas y de relativa insonoridad, de procenios y orquestas ${ }^{39}$.

Esta sentencia llama la atención, porque, hasta donde sabemos, Barrios fue el primer concertista no español de cierto reconocimiento que visitó nuestro país en las primeras décadas del siglo XX. Esto pudo haber generado una disposición diferente del público y la crítica hacia su trabajo, a pesar de que se le trató de vincular de igual forma a la cultura hispánica: “[e]l señor Barrios no es propiamente español; pero desciende de ellos, como de ellos descendemos todos los americanos. No es raro, pues, que sienta por la guitarra el amor suficiente para consagrarle su vida" 40 .

Otra dura crítica que se le realizó fue el haber tocado con cuerdas metálicas. Se especula que Barrios utilizó este material al inicio de su carrera porque su disponibilidad era mayor. Sin embargo, en una etapa posterior de su trayectoria, y con mejores posibilidades de adquisición de cuerdas de tripa, pudo haberlas dejado y no lo hizo. Las razones de esta decisión también quedan en el ámbito de lo especulativo: puede haber sido una cuestión de gusto o simplemente una costumbre ya arraigada (Hoke 2013). Más allá de eso, esta decisión fue siempre un antecedente controversial a la hora de evaluar la interpretación de Barrios. Para algunos, las cuerdas metálicas provocaban un sonido superior, brillante y de mayor proyección, mientras que para otros era algo inaceptable, pues no se respetaban las tradiciones del instrumento (Hoke 2013).

Este último fue el tenor de la crítica chilena, pues si bien se señaló que su ejecución ganó en potencia y claridad, se lamentó, a su vez, el haber perdido el carácter tradicional del instrumento, por lo que " $[\ldots]$ más que guitarra parece, en ciertos momentos de trémulo sostenido, un mandolino, el más insípido y más anti-artístico de todos los instrumentos musicales" 41 .

En esa misma línea, nos llama la atención el no haber encontrado, en la revisión de documentos, alguna mención a la adición del traste veinte en la primera cuerda que Barrios incorporó en algunas de sus guitarras (Hoke 2013). Tal vez sea porque en nuestro país no utilizó uno de estos instrumentos, ya que no vemos en el programa de concierto (ver Anexo 3) la inclusión de la obra Un sueño en la floresta, la que requiere de esta adición de traste. Pese a esto, consideramos importante señalarlo, sobre todo en vista del contexto de cambios en la construcción del instrumento que hemos discutido.

También se percibe el impacto y condicionamiento que provocó en el medio local Llobet con sus conciertos el año anterior. A partir de ese hito, creemos que la idea de la guitarra clásica y de su sonido se configuró de una manera determinada, provocando que los juicios y valoraciones posteriores fueran basadas en esa comprensión de la realidad. Por esta razón, se ocupó una metáfora un tanto burda y exagerada para describir a Barrios, al decir que el músico "es una especie de Llobet al alcance de los niños"42.

Al parecer no se valoró en propiedad el aporte al repertorio guitarrístico que estaba proponiendo Barrios, quien ejecutó en sus conciertos en Santiago varias de sus propias composiciones (ver Anexo 3). Como hemos visto, es posible que no existiera en los críticos

39 Loris, "Concierto Barrios", El Diario Ilustrado XII/4105 (12 de agosto, 1913), p. 4.

40 Loris, "Concierto Barrios", El Diario Ilustrado XII/4105 (12 de agosto, 1913), p. 4.

41 Loris, "Concierto Barrios", El Diario Ilustrado XII/4105 (12 de agosto, 1913), p. 4.

42 Loris, "Concierto Barrios", El Diario Ilustrado XII/4105 (12 de agosto, 1913), p. 4. 
una preocupación por juzgar la calidad de la música misma, sino en determinar si el guitarrista alcanzaba ese estatus de "artista" con el que anteriormente se había catalogado a otros exponentes como Manjón y Llobet. También puede que el sonido proyectado por la guitarra con cuerdas metálicas haya sido un elemento que condicionó el juicio de sus presentaciones en Santiago. Todo indica que Barrios no fue considerado un gran artista, a pesar de ser un guitarrista que se encontraba en un alto momento de su carrera ${ }^{43}$. Esto nos abre la posibilidad de revisar el impacto de su visita desde otras dimensiones.

\section{CONSIDERACIONES FINALES}

La revisión de prensa realizada nos informa de una interesante actividad guitarrística en los albores del siglo XX. Con relación a la idea arraigada -seguramente por desconocimiento-, de que existía escaso movimiento en esta época, resulta notable comprobar, a modo de ejemplo, que en julio de 1918 haya sido posible asistir en Valparaíso a un concierto de Manjón y Llobet en una misma semana. Es destacable verificar, entonces, que algunos de los artistas de mayor fama de la época brindaron conciertos y tuvieron estadías más o menos prolongadas en nuestro país.

Corroboramos, asimismo, la vigencia durante todo este período de los mismos discursos acerca de la categoría social y artística de la guitarra; así como del relato adanista y de transformación/redención del instrumento. También, la predominancia de una visión y un modo de valorar la técnica de ejecución musical que evidentemente condicionaba la apreciación de los conciertos de estos guitarristas extranjeros.

Con relación a la apreciación negativa de la guitarra en la época, a partir de la lectura de las fuentes revisadas creemos identificar tres razones. En primer lugar, su vinculación con segmentos populares o rurales de la población, de los que la elite pretendía diferenciarse. En segundo lugar, el tipo de repertorio que, por lo general, interpretaba la guitarra en el contexto local era considerado menor en comparación a la música docta europea. Por último, no formaba parte de los instrumentos que componían la orquesta sinfónica, lo que constituía un dato importante a la hora de definir su categoría artística.

El análisis de estos guitarristas no debiera pasar por alto también el contexto de transformación en los modelos de construcción de guitarras. Creemos que cada guitarrista, con las innovaciones aplicadas a su propio instrumento, proponía un ideal sonoro particular que se diferenciaba del resto. Esto nos parece relevante, frente a la posibilidad de que la apreciación negativa del sonido de Barrios haya estado mediada por esta estandarización de un ideal sonoro que, suponemos, estableció Llobet en nuestro país. Esto es un antecedente ineludible para realizar una revisión más profunda a futuro.

Del mismo modo, la circulación de guitarristas extranjeros acá informada es un antecedente valioso que contribuye al conocimiento del contexto guitarrístico chileno de la época, pero claramente necesita ser complementado con otros aportes. En estudios posteriores deberían sumarse a este análisis la actividad de otros artistas extranjeros y nacionales. Pensemos, por ejemplo, en Antonio Alba, Francisco Rubí, Carlos Pimentel, Joaquín Zamacois o José Pavez. Más aún, cuántos guitarristas y profesores que desarrollaron actividades en regiones y que permanecen aún en el desconocimiento.

43 Prueba de esto es que justamente por esa fecha Barrios comenzó su carrera discográfica, grabando en Buenos Aires, entre 1913 y 1914, diecisiete discos para la compañía Atlanta Artigas. Véase Hoke 2013: 14. 
Hasta donde sabemos, los "herederos" chilenos de esta etapa preinstitucional de la guitarra clásica, fundamentalmente José Pavez Rojas, nunca llegaron a formar parte de la institucionalidad de la enseñanza que se instauraría a partir de 1938. Por esta razón, su influencia como formadores y referentes de nuevas generaciones de artistas locales, al menos en ese ámbito, parece haber sido escasa y marginal. Sin embargo, resulta importante conocer en mayor detalle el vínculo de Pavez con Llobet, porque -de confirmarse la supuesta relación que une a estos artistas- significaría, entre otras cosas, que el guitarrista local conoció de cerca toda la escuela que se estaba desarrollando en Cataluña, de forma que sería "[...] uno de los primeros músicos guitarristas con la formación de la nueva escuela técnica de Tárrega [...]” (Uribe 2003: 126).

A partir de la lectura de prensa exclusivamente, no es posible conocer las razones que impulsaron la realización de los conciertos de Manjón, Llobet y Barrios, cómo se gestaron, ni qué institución fue patrocinante. Tampoco las motivaciones que tuvo Manjón para residir en nuestro país al menos un par de años. Suponemos, sin embargo, que las causas se deben vincular fundamentalmente con el interés artístico de realizar giras, difundir el repertorio guitarrístico y desarrollar una carrera como concertistas internacionales. Estas interrogantes, que por cierto son importantes de conocer, representarían otro escrutinio en la revisión de la actividad de estos guitarristas.

Asumimos que es indispensable complementar los datos acá presentados con otras fuentes, como cartas y memorias, así como con otras perspectivas de análisis que permitan comprender de mejor forma el contexto informado. Además, resultaría adecuado incorporar enfoques particulares para el análisis de cada guitarrista que no se agoten en una revisión hemerográfica. De esta forma, estaremos en condiciones de alcanzar y proponer conclusiones mayores.

\section{BIBLIOGRAFÍA}

AcHondo, Luis

2015 "Mitos, ideas y agencia en la modernización de la guitarra de Andrés Segovia". Tesis de Magíster. Santiago: Pontificia Universidad Católica de Chile.

Calisto, Jaime

1996 "Evolución de la enseñanza de la guitarra en Chile". Memoria de pregrado inédita. Santiago: Escuela Moderna de Música.

Chiantore, Luca

$2001 \quad$ Historia de la técnica pianistica. Madrid: Alianza Editorial, S.A.

Escande, Alfredo

1996 La escuela de Carlevaro. Conferencia en Primer Festival Internacional de Guitarra. Cabildo de Montevideo, Uruguay. http:/ / www.elguitarrista.net/articulos/la_escuela_de_carlevaro. html [acceso: 12 de junio, 2020].

FERNÁNDEZ, EduARdo

2000 Técnica, mecanismo, aprendizaje. Una investigación sobre llegar a ser guitarrista. Montevideo: ART Ediciones.

Fubini, ENRICO

2004 Estética de la música. Madrid: Antonio Machado Libros.

González, Juan Pablo y Claudio Rolle

2005 Historia social de la música popular en Chile, 1890-1950. Santiago: Ediciones Universidad Católica de Chile.

HERnÁNDEZ, FABián

2010 "La obra compositiva de Emilio Pujol (1886-1980): Estudio comparativo, Catálogo y edición crítica”. Tesis doctoral. Barcelona: Universidad Autónoma de Barcelona. 
Hoke, Justin

2013 "The guitar recordings of Agustín Barrios Mangoré: an analysis of selected works performed by the composer". Tesis doctoral, The Florida State University College of Music.

Honour, Hugh

1981 El romanticismo. Madrid: Alianza.

Johansson, Mats

2014 "On the relationship between technique and style: the case of the violin". Music Education Research, 17/2, pp. 127-140. DOI: https://doi.org/10.1080/14613808.2014.930114

Milanca Guzmán, Mario

2000 "La música en el periódico chileno 'El Ferrocarril' (1855 -1865)”, Revista Musical Chilena, LVI/193 (enero-junio), pp. 17-44. DOI: http://dx.doi.org/10.4067/S0716 $-27902000019300002$

Mosquera, Gerardo

2010 Caminar con el diablo: textos sobre arte, internacionalismo y culturas. Madrid: Exit.

Otero, ANA María

2010 Documentos musicales en la prensa de Mendoza. Ediciones Biblioteca Digital UNCuyo. https:// bdigital.uncu.edu.ar/4030 [acceso: 12 de junio, 2020].

Phillips, RoberT

2002 "The influence of Miguel Llobet on the pedagogy, repertoire, and stature of the guitar in the twentieth century". Tesis doctoral. Miami: Universidad de Miami.

PRAT, DOMINGO

1934 Diccionario de guitarristas. Buenos Aires: Casa Romero y Fernández.

Pujol, EMilio

1934 El dilema del sonido en la guitarra. Buenos Aires: Casa Romero y Fernández.

RAmos, Ignacio

2005 Historia de la guitarra y los guitarristas españoles. España: Editorial Club Universitario.

2009 “Antonio Jiménez Manjón en Madrid (1913)”. Disponible en http://www.ahisvi.es/ Articulos/13.pdf [acceso: 12 de junio, 2020].

2012 "La guitarra clásica en la ciudad de Alicante (1875-1936)". Roseta. Revista de la Sociedad Española de la Guitarra, 7, pp. 20-50.

RoDríguez, EugENiA

2001 "Liliana Pérez Corey (1917-1990). Pionera en la enseñanza de la guitarra en Chile", Resonancias, V/8 (mayo), pp. 31-43.

Soto, PABLO

2018a "La técnica de la guitarra clásica en el Chile del siglo XX. Aportes para una historia crítica". Tesis doctoral. Santiago: Pontificia Universidad Católica de Chile. Disponible en https:// repositorio.uc.cl/handle/11534/22090 [acceso: 20 de noviembre, 2020].

2018b "Apuntes sobre la estadía en Chile de Albor Maruenda. Primer profesor de la cátedra de guitarra del Conservatorio Nacional”, Neuma, Revista de Música y Docencia Musical, XI/2, pp. 96-111.

Uribe, Cristhian

2003 "Cien Años de Guitarra en Chile: de los Salones Criollos a la Sala de Conciertos (18801980)". Tesis doctoral. Oviedo: Universidad de Oviedo.

Vera, Alejandro

2014 "Music, Eurocentrism and Identity: The Myth of the Discovery of America in Chilean Music History". Advances in Historical Studies, 3, pp. 298-312. DOI: http://dx.doi.org/10.4236/ ahs.2014.35024 
2016 "La música entre escritura y oralidad: la guitarra barroca, el guitarrón chileno y el canto a lo divino". Revista Musical Chilena, LXX/225, pp. 9 - 49. DOI: http://dx.doi.org/10.4067/ S0716-27902016000100001.

\section{Fuentes hemerográficas}

El Diario Ilustrado, Santiago, 1913.

El Mercurio, Santiago, 1912.

El Mercurio de Valparaíso, 1893, 1912 y 1918.

El Noticiero Español. Periódico semanal independiente. Dedicado a la colonia española, Santiago, 1893.

El Sur, Concepción, 1918.

La Mañana, Talca, 1918.

Las Últimas Noticias, Santiago, 1918.

Revista Música, Santiago, 1920 y 1922.

Revista Sucesos de Valparaíso, 1912.

\section{ANEXO 1. PROGRAMA DE CONCIERTO DE ANTONIO JIMÉNEZ MANJÓN 22 de diciembre de 1893. Valparaíso, Chile}

\section{Primera Parte}

Piano $^{44}$

Guitarra y piano

Guitarra sola
Fantasía Impromptu

Primer tiempo de concierto op. 192

Andante y variaciones

Rondó en La menor ${ }^{45}$

Un recuerdo a mi patria

Polonesa

Adajio de la Sonata 14

Célebre Fandango

\author{
Chopin \\ Herz \\ Purcel \\ Aguado \\ Manjón \\ Arcas \\ Beethoven \\ Manjón
}

\section{Segunda Parte}

Piano

Guitarra sola

Rapsodia española

Andante y alegro de la $2^{\circ}$ gran Sonata en Do

Nocturno

Sor

Momento appasionato

Guitarra y piano

Jota

Coste

Schumann

Manjón

El Mercurio de Valparaíso, 21 de diciembre, 1893.

${ }^{44}$ Piano interpretado por Rafaela Salazar, esposa de Manjón.

45 Pensamos que debe haber un error en la publicación del diario, ya que el Rondó en Lam es una obra solista para guitarra, y acá se dice que es interpretada también en piano. 


\section{ANEXO 2. PROGRAMA DE CONCIERTO DE MIGUEL LLOBET} 22 de julio de 1918. Valparaíso, Chile

\section{Primera Parte}

Menuet

Sor

Preludio

Andante

Mozart

Recuerdos de la Alhambra

Tárrega

Danza Española

Granados

Nocturno

Chopin

\section{Segunda Parte}

Pizzicato

Tárrega

Dos Mazurkas

Alborada

Mazurka

Bufaletti

Granada

Albéniz

Gran Jota

Arcas

El Mercurio de Valparaíso, 23 de julio, 1918, p. 3.

\section{ANEXO 3. PROGRAMA DE CONCIERTO DE AGUSTÍN BARRIOS}

Santiago el 11 de noviembre de 1913

\section{Primera Parte}

Fantasía en Mi

Viñas

Gran vals de concierto

Barrios

Estudio N.12

Aguado

Moraima, fantasía morisca

Espinoza

Capricho andaluz

Barrios

\section{Segunda Parte}

Concierto en La

Arcas

Trovatore miserare

Verdi

Divagación chopiniana

García Tolsa

Gavota clásica

Czibulka

Rapsodia americana

Barrios

Dieciséis variaciones sobre motivos de Jota

Barrios

El Diario Ilustrado, Santiago, 11 de noviembre, 1913. 